\title{
THE STRUCTURE OF $\mathrm{Yb}\left(\mathrm{ND}_{3}\right)_{5,7}$
}

\author{
C. NÓLDEKE, P. DAMAY*, W. PRESS, F. LECLERCQ*, W. LIPPIK and M. FOUKANI* \\ Institut für Experimentalphysik der Universität Kiel, Leibnizstrasse 19, D-2300 Kiel, Germany \\ *URA 253 du CNRS, HEI, 13 rue de Toul, F-59046 Lille cedex, France
}

\begin{abstract}
Neutron powder diffraction was performed on five $\mathrm{Yb}\left(\mathrm{ND}_{3}\right)_{x}$ samples prepared with $x=5.5,5.7,5.96 .0$ and 6.3. Two different low temperature $(2 \mathrm{~K})$ structures are observed; one (i) mainly showing peaks of a bcc lattice with $a_{0}=9.076 \AA$ but also weak sc peaks, the other (ii) presenting bcc peaks only with $a_{0}=9.111 \hat{A}$. At $17 \mathrm{~K}$ the sc peaks in (i) vanish, which indicates the presence of an order- disorder phase transition. For the orientationally disordered structure (ii). $\mathrm{Yb}\left(\mathrm{ND}_{3}\right)_{5.7}$, showing more disorder at low temperature, a structure refinement with cubic harmonic functions is given.
\end{abstract}

1. Introduction.

Neutron scattering experiments on $\mathrm{Ca}\left(\mathrm{ND}_{3}\right)_{x}$ and $\mathrm{Ca}\left(\mathrm{NH}_{3}\right)_{x}$ with $x$ ranging from 5.0 to 6.5 have provided a series of interesting results $/ 1,2,3,4 \%$. One is the frozen-in orientational disorder of the ammonia groups at low temperatures $/ 2 \%$. This indicates the presence of a rather weak orientational potential, for which further evidence is given by the observation of nearly free uniaxial quantum rotations of the $\mathrm{NH}_{3}$ groups $/ 3 /$.

From a similar study on $\mathrm{Yb}\left(\mathrm{ND}_{3}\right)_{x}$ and $\mathrm{Yb}\left(\mathrm{NH}_{3}\right)_{x}$ compounds, we expected to learn about the effect of lon substitution $U_{p}$ to now, only one sample $\left(\mathrm{Yb}\left(\mathrm{NH}_{3}\right)_{5.5}\right)$ showed quantum exitations of the ammonia groups $/ 5 /$. This can be related to the results of the structure determination presented in this paper.

\section{Experimental}

Samples were prepared as described in ref. 2, the last step before sealing off the container being the addition of the proper amount of $\mathrm{ND}_{3}$ to the powdered $\mathrm{Yb}$. This way the nominal values for the stoichiometry $x$ were obtained. Five samples were chosen for the experiments, three in a niobium can $(x=5.5,5.7,5.9)$, the other two $(x=6.0,6.3)$ in a quartz cell.

Data were taken with the neutron powder diffractometer D2B at the ILL in Grenoble with $\lambda=1.594 \AA$. After cooling down to $2 \mathrm{~K}$ within about an hour, several powder patterns were taken with a stepwise increase of temperature up to $180 \mathrm{~K}$ during ten hours. At $2 \mathrm{~K}$ measurements with different sample orientations were performed to ensure the absence of preferred orientation. 


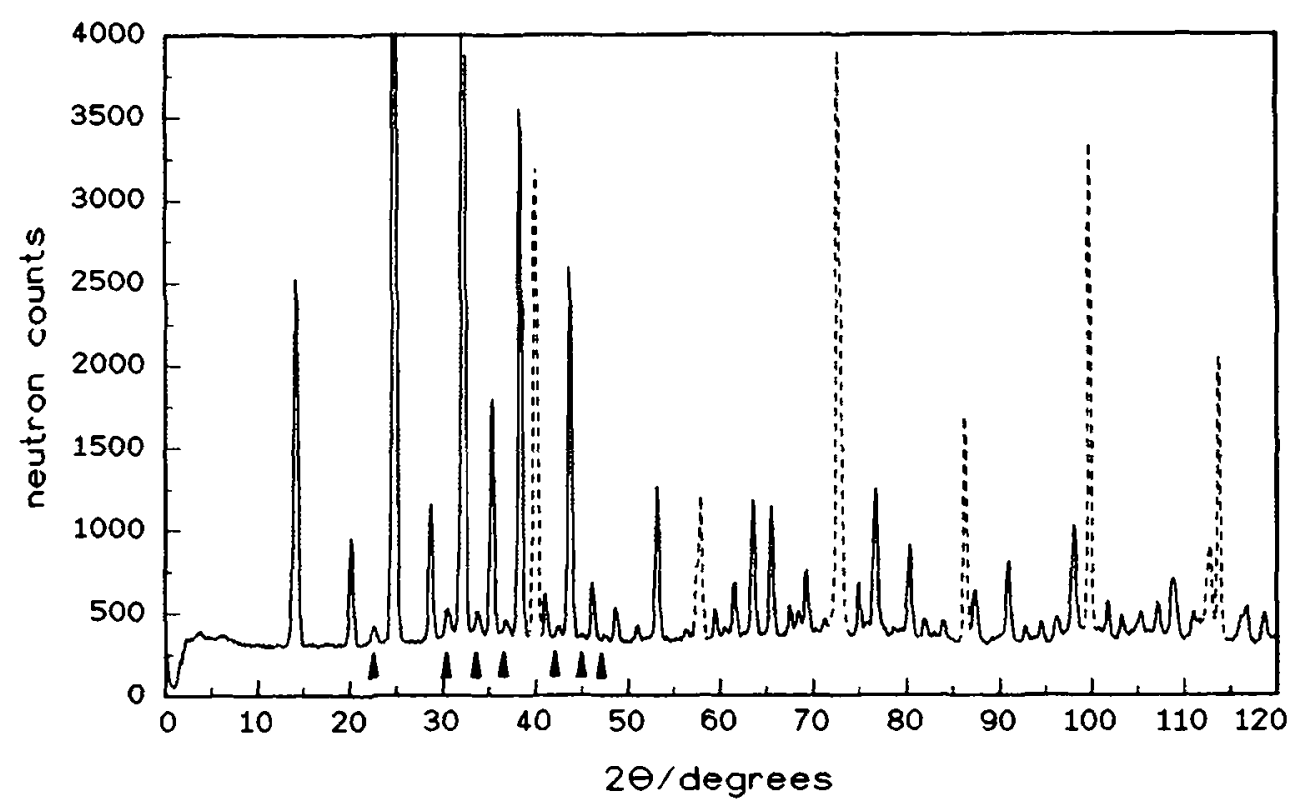

Figure 1. Powder pattern of $\mathrm{Yb}\left(\mathrm{ND}_{3}\right)_{5.9}$ at $T=2 \mathrm{~K} / \lambda=1.594 \AA$, measured at the high resolution powder diffractometer $\mathrm{D} 2 \mathrm{~B}$. The peaks which originate from the niobium sample container and cryostat are drawn with dashed lines, the arrows mark the simple cubic peaks.

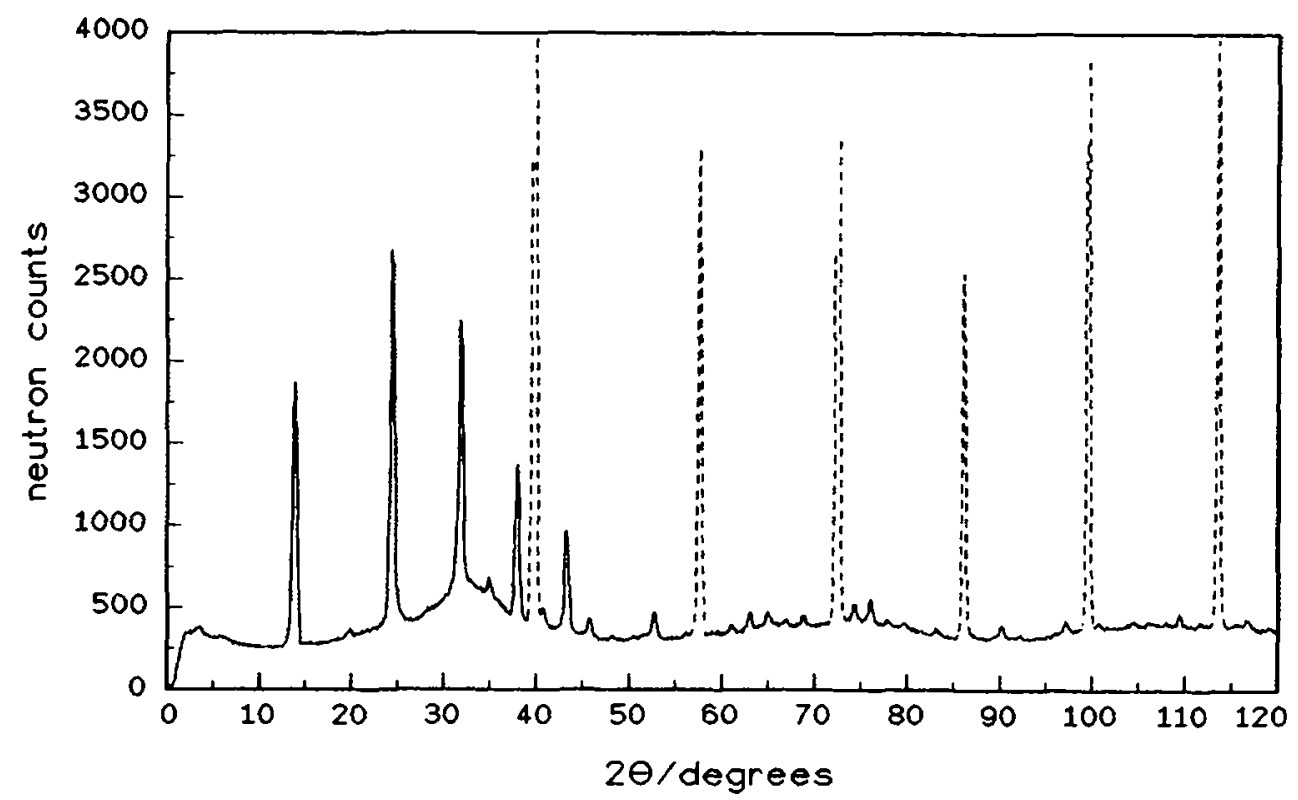

Figure 2. Powder pattern of $\mathrm{YB}\left(\mathrm{ND}_{3}\right)_{5.7}$ at $T=2 \mathrm{~K}, \lambda=1.594 \AA$. The container peaks are marked the same way as in figure 1 . Note the vanishing peaks for larger angles and the diffuse scattering. 


\section{Results}

a) First evaluation of the $2 \mathrm{~K}$ powder patterns

Apart from a scale factor the observed intensities of the $\mathrm{Yb}\left(\mathrm{ND}_{3}\right)_{X}$ peaks are identical for the samples with $x=5.5,5.9,6.0$ and 6.3. The last two samples showed additional peaks from crystalline $\mathrm{ND}_{3}$ which indicates an incomplete reaction. A consequence are lower values for the stoichiometries than the nominal values $x$. The powder pattern of the $x=5.9$ compound is shown in figure 1. This sample is discussed as a representative of all four in the following. The different powder pattern of $\mathrm{Yb}\left(\mathrm{ND}_{3}\right)_{5.7}$ is shown in figure 2 .

Comparing both patterns a common feature are high intensity peaks at low angles only, indicating the presence of disorder. As the intensities decrease faster with increasing angle for the $x=5.7$ compound, this particular sample shows more disorder, in agreement with the larger amount of diffuse scattering.

b) Lattice type / cell parameters

The peaks of the $\mathrm{Yb}\left(\mathrm{ND}_{3}\right)_{5.7}$ sample (fig. 2) all could be indexed as bcc with $\mathrm{a}_{0}=9.111$ (1) $\AA$. The other powder pattern (fig. 1) shows some small additional peaks which give a simple cubic lattice with $a_{0}=9.076$ (1) $\AA$. As the relative intensities tell, the deviations from a bcc lattice with the same lattice constant are small. Above $17 \mathrm{~K}$ the superlattice peaks are no longer observed. The temperature dependence of the lattice constants is given in figure 3 . Remarkable features are the anomaly around $80 \mathrm{~K}$ for the $x=5.7$ sample and the indication of a sigmoidal shape around $40 \mathrm{~K}$ in the other case.

\section{c) Diffuse scattering}

Between $30 \mathrm{~K}$ and $45 \mathrm{~K}$ we observe a considerable increase in the diffuse scattering for the sample with $x=5.9$ (fig. 4). The $Q$ dependence at $45 \mathrm{~K}$ is similar to the one of the other sample at $2 \mathrm{~K}$, which changes little with temperature.

\section{d) Structure refinement}

So far no acceptable model for the structure with superlattice peaks $\left(\mathrm{Yb}\left(\mathrm{ND}_{3}\right)_{5.9}\right)$ has been found. For the structure refinement of $\mathrm{Yb}\left(\mathrm{ND}_{3}\right)_{5.7}$ we used an approach wtih cubic harmonics. This description is especially suitable in the present case of an orientationally disordered molecular crystal. The rotational form factor $/ 6 /$ is given by:

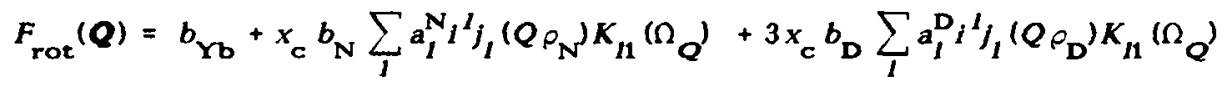

Possible deviations in the stoichiometry from six are taken into account by the coefficient $x_{c}$. $\Omega_{Q}=\left(\varphi_{Q}, \Theta_{Q}\right)$ are the polar angles of $Q$ in the cubic frame, the $j_{l}(Q \rho)$ denote spherical Bessel functions of the order $l$ and the $K_{I}\left(\Omega_{Q}\right)$ are cubic harmonics of the order $I=0,4,6$, 8 and 10. The coefficients $a_{l}^{N}$ and $a_{1}^{D}$ are coupled via the ammonia geometry by (adapted from ref. 7):

$$
a_{1}^{D}=P_{1}(\cos \vartheta) a_{1}^{N} \quad \text { (2) } \quad \text { with } \quad \cos \vartheta=\frac{\sqrt{\rho_{D}^{2}-r_{D}^{2}}}{\rho_{D}}
$$




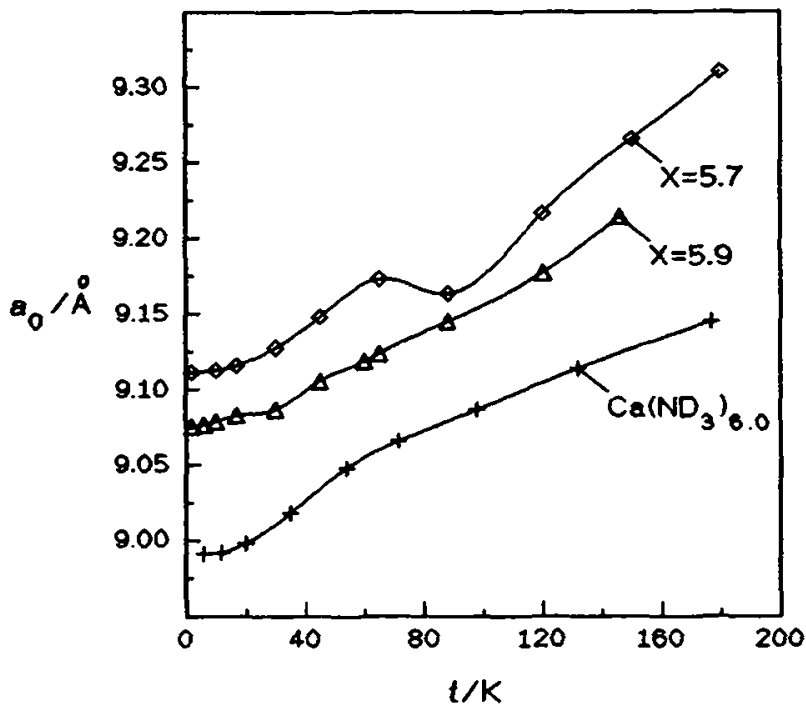

Figure 3. Temperature dependence of the lattice constants. The values for $\mathrm{Ca}\left(\mathrm{ND}_{3}\right)_{6.0}$ are given for comparison. The lines are drawn as guides to the eye only.

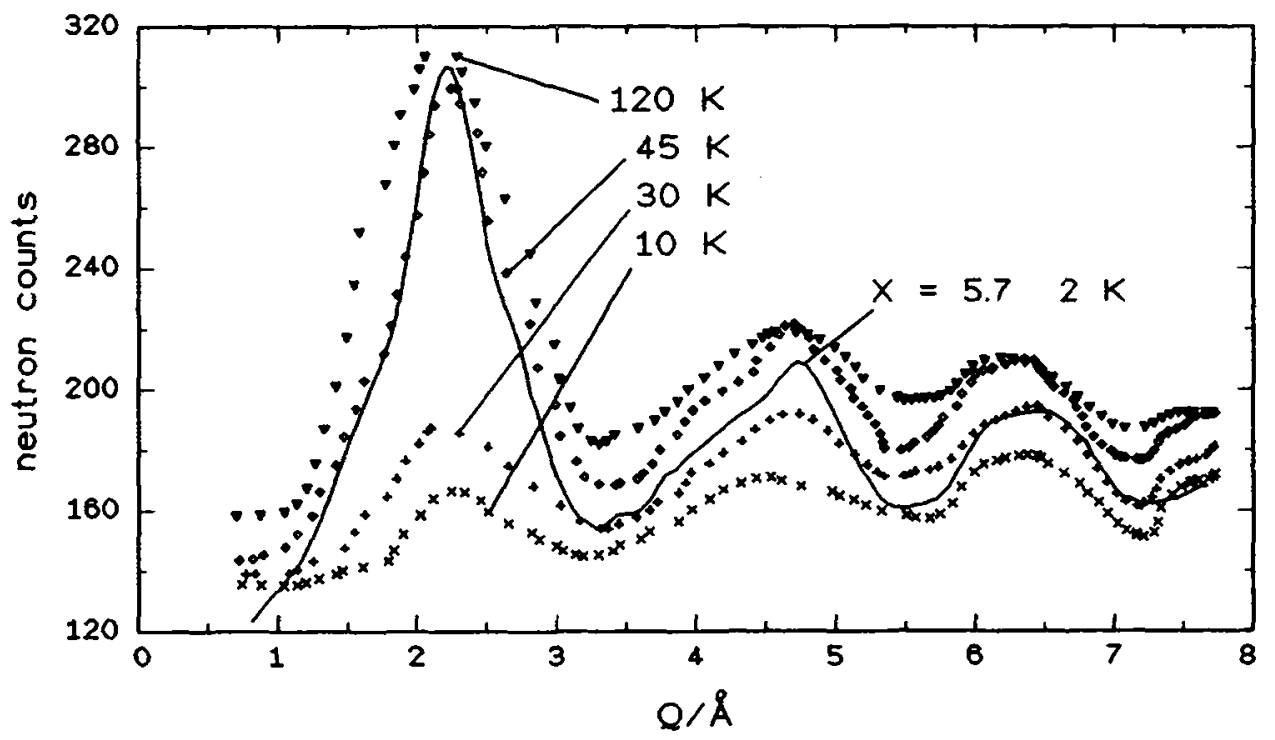

Figure 4. Diffuse scattering of $\mathrm{Yb}\left(\mathrm{ND}_{3}\right)_{5.9}$. The $Q$ dependence is given for four different temperatures to show the increase above $30 \mathrm{~K}$. The line gives the diffuse scattering of $\mathrm{Yb}\left(\mathrm{ND}_{3}\right)_{5.7}$ at $2 \mathrm{~K}$. 
where $P_{1}(\cos \vartheta)$ denotes the Legendre polynomials. $\rho_{\mathrm{D}}$ the $\mathrm{Yb}-\mathrm{D}$ distance and $r_{\mathrm{D}}$ the distance of the deuterium atom from the symmetry axis of the ammonia molecule.

The parameter values obtained from the least squares fit to the powder pattern are given in the table. Adding the contribution of higher orders of cubic harmonics did not significantly improve the fit. The value of $x_{c}$ was expected to be about the same as the nominal value of the stoichiometry $x=5.7 \pm 0.1$ The result of the fit gives a strikingly different value of $x_{c}=7.0$. which is not within the error estimated from preparation. As can be seen fom the table $P_{N}$ and $P_{D}$ were not coupled in the fit. The heigth of the ammonia tetrahedon calculated from these values via

$$
h_{\mathrm{ND}_{3}}=\sqrt{\rho_{\mathrm{D}}^{2}-r_{\mathrm{D}}^{2}}-\rho_{\mathrm{N}}
$$

gives $h_{\mathrm{ND}_{3}}=0.46 \AA$ which is close to the nominal value of $0.43 \AA / 4 /$.

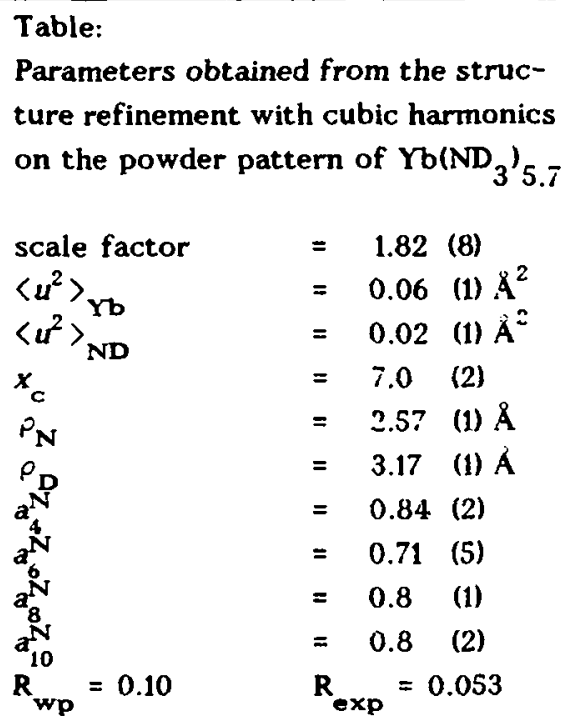

The structure refinement has been also performed for a series of models, which place orientationally disordered ammonia in positions of special symmetry. A model with ammonia coordinated octahedrally to the $\mathrm{Yb}$ did not give any acceptable agreement. Allowing for ammonia in tetrahedal positions also, resulted in $R$ values close to the fit with cubic harmonics. This is the same model which has been used in the case of $\mathrm{Ca}\left(\mathrm{ND}_{3}\right)_{5.7} / 2 /$. A further improvement was achieved when a fraction of the ammonia in tetrahedal positions was oriented with the nitrogen atoms pointing outward. In both cases the values obtained for $x_{c}$ were about 7 .

\section{Discussion}

\section{a) Stoichiometry}

In vapor pressure experiments the stoichiometry of solid $\mathrm{Yb}\left(\mathrm{NH}_{3}\right)_{\mathrm{x}}$ was determined by removal of ammonia from a $\mathrm{Yb} / \mathrm{NH}_{3}$ solution. The results give a composition close to 6.5./8,9/. That questions the value $x_{c}=7.0$ obtained from the structure refinement; one will have to check carefully the correlations between this parameter, the Debye-Waller factors and the coefficients of the cubic harmonics.

The samples prepared with $x=5.5,5.9,6.0$ and 6.3 show similar diffraction patterns, which suggests an identical composition in all four cases. Since additional ND3 peaks are found only for the 6.0 and 6.3 samples and not for the 5.9, nor Yb peaks for the 5.5 or 5.7 sample, an open problem remains, which will require further efforts. 
b) order- disorder transition

The disappearence of the sc peaks at $17 \mathrm{~K}$ is a clear indication of an order- disorder phase transition. It explains the increase of the diffuse scattering for the $x=5.9$ sample, and the sigmoidal shape of the temperature dependence of the lattice parameter. Basically the same type of disorder freezes in for the $x=5.7$ sample. As a result of the structure refinement this is understood as combination of positional disorder of $\mathrm{ND}_{3}$ around the $\mathrm{Yb}$ ion with the polar axis pointing at the $\mathrm{Yb}$, and an orientational disorder of the ammonia groups around this axis.

\section{Conclusion}

The results obtained in this study are in agreement with the present knowledge about solid metal ammonia compounds. The orientational order- disorder phase transition is observed for the other compounds too $/ 1 /$. As in the case of $\mathrm{Ca}\left(\mathrm{ND}_{3}\right)_{x}$ the stoichiometry determines wether the disorder freezes in or not $/ 2 /$. The discrepancy of the nominal values for the stoichiometry from preparation and the ones obtained from structure refinement for the Ca compounds again shows up. This time they are more significant due to the larger scattering length of the $\mathrm{Yb}\left(\mathrm{b}_{\mathrm{b}}=1.2410^{-12} \mathrm{~cm}\right.$ vs. $\left.\mathrm{b}_{\mathrm{Ca}}=0.4910^{-12} \mathrm{~cm}\right)$.

Within experimental errors earlier results from $x$-ray powder diffraction are reproduced (at $198 \mathrm{~K}$ : bcc $9.30 \AA$, distance $\mathrm{Yb}-\mathrm{N} 3.0 \pm 0.3 \AA / 10 /$ ).

The anomaly of the lattice constant of the $x=5.7$ compound at $80 \mathrm{~K}$ is not yet explained. Further experimental evidence, with different cooling and heating rates ( $T_{g l a s s}$ ?) would be helpful to find an convincing interpretation.

Acknowledgements

Part of this work was supported by the Bundesminister fur Forschung und Technologie (BMFT) under Grant No. PR2KIE. Instrument time, technical and scientific support was provided by the Institut Laue Langevin, Grenoble, France, which is greatfully acknowledged.

\section{References}

/1/ W. S. Glaunsinger, R. B. von Dreele, R. F. Marzke, R. C. Hansen, P. Chieux, P. Damay and R. Catterall, J. Phys. Chem. 88, 3860 (1984)

/2/ W. Press, P. Damay, F. Leclercq and P. Chieux, J. Chem. Phys. 91 (2), 1167 (1989)

/3/ P. Damay, F. Leclercq, A. J. Dianoux and W. Press, Springer Proc. in Physics, Vol. 37 ed. D. Richter, p. 266 (1989)

14/ F. Leclercq, P. Damay and P. Chieux, J. Phys. Chem. 9s , 7300 (1990)

/5/ C. Nöldeke et al., unpublished results

/6/ W. Press and A. Hüler. Acta Crstallogr. A 35, 876 (1979)

$17 /$ M. Bée. Quasielastic Neutron Scattering, p. 253-257 (1988)

/8/ R. Hagedorn and J. P. Lelieur, J. Phys. Chem. 85, 275 (1981)

19/ M. Foukani, diplome d'etudes approfondies sciences des materiaux, HEI, Lille (1990)

/10/ N. Mammano, in Metal ammonia Solutions, edited by J. Lagowski, M. Sienko, London (1970) 